\title{
Considerations for Sensitivity Analysis, Uncertainty Quantification, and Data Assimilation for Grid-to- Rod Fretting
}

\author{
Michael Pernice
}

October 2012

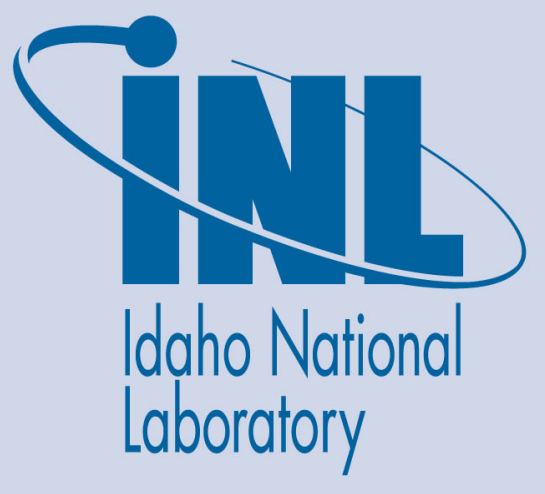

The INL is a U.S. Department of Energy National Laboratory operated by Battelle Energy Alliance 


\title{
Considerations for Sensitivity Analysis, Uncertainty Quantification, and Data Assimilation for Grid-to-Rod Fretting
}

\author{
Michael Pernice
}

October 2012

\begin{abstract}
Idaho National Laboratory
Idaho Falls, Idaho 83415
\end{abstract}

http://www.inl.gov

Prepared for the

U.S. Department of Energy

Office of Nuclear Energy

Under DOE Idaho Operations Office

Contract DE-AC07-05ID14517 


\title{
Considerations for sensitivity analysis, uncertainty quantification, and data assimilation for grid-to-rod fretting
}

\author{
Michael Pernice \\ Center for Advanced Modeling and Simulation \\ Idaho National Laboratory, Idaho Falls, ID 83415
}

INL/EXT-2012-27267

October 8, 2012

\begin{abstract}
Grid-to-rod fretting is the leading cause of fuel failures in pressurized water reactors, and is one of the challenge problems being addressed by the Consortium for Advanced Simulation of Light Water Reactors to guide its efforts to develop a virtual reactor environment. Prior and current efforts in modeling and simulation of grid-to-rod fretting are discussed. Sources of uncertainty in grid-to-rod fretting are also described.
\end{abstract}

\section{Introduction}

The Consortium for Advanced Simulation of Light Water Reactors (CASL) is developing advanced modeling and simulation capabilities for light-water reactors (LWRs). CASL's principal goal is to develop predictive simulations of LWR performance that couple state-of-the-art models for fuel performance, neutron transport, thermal-hydraulics, and structural dynamics with existing system/safety analysis tools. This effort is guided by a collection of challenge problems whose solution will lead to improved operational efficiency and/or increased safety of LWRs

One of the challenge problems that CASL is addressing is grid-to-rod fretting (GTRF), which is the leading cause of fuel failures in pressurized water reactors (PWRs). Improved understanding of GTRF can help to reduce capital and operating costs per unit energy by allowing power uprates and increased cycle length, and to reduce the volume of generated waste by allowing higher fuel burn-up.

The principle driver for GTRF is flow-induced vibration that is due to turbulent flow of the coolant. In a fuel assembly, a fuel rod is held in place by friction 
forces between the fuel rod and springs and dimples in several spacer grids that are part of the assembly. Initially, the flow-induced vibrations cause small relative motions between the fuel rod and the spacer grids. Over the lifetime of the fuel assembly, gaps between the fuel rod and its supports can appear, due to deformation of the spacer grids caused by radiation creep and growth. The amplitude of the vibrations increases as a result of these gaps. Movement of the fuel rod within the assembly can lead to mechanical wear of the fuel cladding at locations where the fuel rod is in contact with the spacer grids. Structural and chemical changes in the cladding and spacer grid materials can also affect the wear rate. Release of fission gas and thermal expansion of the fuel pellets swell and stress the cladding, corrosion thins the fuel cladding, and hydrogen uptake hardens and embrittles the cladding, increasing the wear rate. Wear scars can increase in depth and eventually cause perforation of the cladding. Simulation of GTRF involves resolving the turbulent flow of the coolant, fluid-structure interaction between the coolant and the fuel assemblies, mechanical contact between the fuel rod and its supports, and modeling of mechanical wear. See [11] and references therein for more information. CASL's simulations of GTRF are focused on determination of the turbulent fluid forces on the fuel rod, prediction of the gap between the fuel rods and spacer grids, vibration of the fuel rod, and wear at the sites where fretting occurs.

Late in the most recent CASL performance period, it was determined that the potential gains of continuing to pursue CASL'S current approach to simulating GTRF did not warrant the effort and resources required. A concurrent effort in validation and uncertainty quantification (VUQ), which is based on CASL's GTRF simulation capabilities, was likewise discontinued. The objective of the VUQ effort was to perform sensitivity analysis and uncertainty quantification of maximum wear depth, using the friction coefficient and fuel rod stiffness as uncertain parameters. This report documents information gained and lessons learned from this VUQ activity, and is organized as follows. Section 2 discusses models and algorithms employed for GTRF, and describes CASL's approach to GTRF simulations. Section 3 discusses sources of uncertainty in GTRF and describes some ways to analyse them. Section 4 draws some conclusions and makes some recommendations for a path forward in uncertainty analysis of GTRF.

\section{Models and Algorithms for GTRF}

The CASL L3:MPO.GTRF.P2.05 milestone focused on improved modeling of materials tribology and wear for GTRF. This effort included an assessment of the available modeling frameworks for GTRF, and identified VITRAN [14, 16] as the only currently available modeling framework for GTRF [5]. VITRAN has been used to reduce the number of experimental scoping studies of fuel assembly designs, to guide choices in new rod support designs, to assess the performance of current designs, and to improve fretting wear margins [15]. However, it should be possible to improve wear depth predictions with higher fidelity physical models; in particular, [5] recommends focusing on improved wear models and detailed 
simulation of the interactions between the cladding and grid supports.

\subsection{VITRAN Analysis}

VITRAN models a single fuel rod as an Euler-Bernoulli beam that features eight spacer grid supports with pinned ends at the top and bottom spacer grid, and nine rod spans. The turbulent fluid pressures are assumed to have the same time variation between any pair of contiguous spacer grids, and are modeled as Gaussian band-limited white noise with a constant power spectral density. The power spectral density is estimated from assembly flow conditions using an experimental correlation developed for turbulent flow in fuel assemblies. The constraining forces at the spacer grids are modeled as point contact forces that are decomposed into normal and tangential components. The normal force is obtained by modeling the grid support as a spring-damper system with a gap, while the tangential force is estimated with a friction law that includes both sticking and sliding regimes. Transitions between these regimes occur when the sliding velocity drops below a specified threshold and when the tangential component exceeds the static friction limit. Normal work rates are estimated from the normal forces and the displacements of the vibrating rod. Wear is determined using Archard's law, which requires normal load and sliding distance as input, and wear volume is accumulated over the course of the simulation. VITRAN is calibrated to data from WEC's VIPER loop test facility [1].

VITRAN addresses uncertainties in initial conditions, boundary conditions, and mechanical properties with a statistical approach that propagates these uncertainties through the simulation. Monte Carlo sampling from prescribed probability distributions of uncertain input parameters is used to generate probability distributions of model responses. Several VITRAN-based analyses of GTRF are available in the literature, notably $[14,16]$. The uncertain parameters in these studies are rod position in the grid (grid misalignment, cell tilt), support stiffness, dynamic and static friction coefficients, and rod-support wear coefficients, with presumed normal distributions with means and standard deviations determined from experimental data. Response quantities included work rates, volumetric wear rates, and scar depths. The main conclusions of these studies were that grid cell clearance and turbulent fluid pressures are key factors in assessing wear risk; grid misalignment and cell tilts are significant, though less important, factors; and assembly bow shape, structural damping, rod stiffness and friction coefficients were all less important factors.

\subsection{CASL Simulation of GTRF}

CASL's efforts to develop predictive capability for GTRF focus on improved determination of fluid pressure boundary conditions on the fuel rod, full threedimensional simulation of the fuel rod vibrations, and higher fidelity modeling of the grid supports [11]. These reflect recommendations made in prior studies for providing boundary conditions to the fuel rod [16] and for wear analysis at contact sites $[5,6]$. 
Currently, fluid pressure boundary conditions are taken from turbulent fluid simulations of a $3 \times 3$ pin geometry of the Westinghouse V5H fuel assembly design. In these simulations, the spacer grids include mixing vanes, which enhance fluid turbulence and heat transfer. The procedure for transferring the fluid pressure load from the fluid mesh to the fuel rod mesh was developed in [13] and is described further in [18]; a verification study of the transfer procedure is given in [2]. Note in particular from [18] that simulations are currently restricted to two spans of the fuel rod, with spacer grids at the middle and both ends of the fuel rod. This simplification is supported by computational evidence that the effects of turbulence induced by a spacer grid rapidly decay downstream in a single span $[4,13,17]$. A further simplification is obtained by replicating the same fluid boundary conditions along each span in the simulation.

The three-dimensional finite element simulation of the vibrating fuel rod employs a viscoplastic model for thermal and irradiation creep from [10]. This constitutive model has been integrated into Sierra, which is a computational framework that facilitates development of multiphysics application software [20]. The explicit time integration capabilities of Adagio are used to calculate fuel rod vibrations, and Sierra's mechanical contact algorithms are used to resolve the interactions between the fuel rod and its supports. Mixing vanes do not play a role in GTRF beyond determining fluid pressures, and so are not included in the solid mechanics simulation. Periodic displacement boundary conditions are imposed on the portion of the spacer grid that is represented in the $3 \times 3$ pin geometry. The friction model from VITRAN [14] and an advanced wear model recommended in [7] are used in CASL's simulations of GTRF.

The use of explicit time integration to simulate GTRF combined with the current spatial resolution of the fuel rod requires a time step of roughly $2.8 \times$ $10^{-8}$ seconds for numerical stability. A half second of simulation time requires nearly 18 million time steps and 8-10 days of computation on 128 processors of currently available high-performance computing systems. In contrast, gaps between the spacer grid and fuel rods only begin to appear after several thousand hours of reactor operation.

\section{Sources of Uncertainty in Modeling GTRF}

VUQ of fretting wear margins can be used to inform design decisions for preventing GTRF or fuel assembly management decisions to reduce the incidence of GTRF-induced fuel failures. There are a large number of potential sources of uncertainty that must be taken into account by uncertainty analysis of GTRF.

Initial conditions must accurately reflect the state of spring pre-loads, which strongly influence the amount of fretting wear [16]. Operating times for occurrence of the first leaks caused by fretting can range from less than 100 to more than 1500 days [9], so there is also a need to accurately determine initial conditions at different stages of plant operation. It is especially important to be able to predict the appearance and size of gaps between the fuel rods and spacer grids. However, there is scant data available on gaps encountered in operating 
reactors [3], so an approach to modeling these gaps and treating them as epistemic uncertainties might be used until such data becomes available. In addition to estimating spatial discretization errors, verification studies of rod vibration should also estimate errors in time, because of the long time scale of fretting damage relative to the time scale of the rod vibrations, and the likelihood of accumulating temporal error across the simulation.

The fluid pressure boundary conditions on the fuel rod are unknown, and as with grid-to-rod gaps there is scant data on this. ${ }^{1}$ This lack of data limits opportunities for data assimilation, and suggests the need to treat these boundary conditions as epistemic uncertainties. Verification studies of turbulent fluid flow for driving GTRF in the geometry described in $\$ 2.2$ [12] suggest that very large simulations, on the order of a billion elements, would be needed to obtain grid-converged solutions. In order to understand the impact of "full core" effects such as cross-flow and jetting, much larger simulations will be needed to determine pressure boundary conditions in full assemblies and cores. Initial/boundary conditions for the coolant flow are also best represented with full core geometry; for example, the studies in [17] treat a subset of a rod bundle that is presumed to be far enough from reactor vessel walls to eliminate cross-flow effects, assume uniform velocity field boundary conditions at the inlet of the fluid computational domain, and stress-free boundary conditions at the outlet of the fluid computational domain, and periodicity at the remaining boundaries of the fluid computational domain. Model uncertainty due to treating only a fraction of the core should be investigated. The location of the fuel assembly in the reactor vessel must also be accounted for, since the amount of fretting has been observed to depend on location [9].

Any uncertainty in fluid load on the fuel rod will propagate to the vibrational behavior of the fuel rod, and will affect wear rates through the shear traction (via the Coulomb friction model) and the tangential velocity in the direction of the shear traction [7]. Approaches for sampling uncertain pressure boundary conditions to propagate these uncertainties through the vibrating rod to the wear model need to be devised. Numerical treatment of fluid-structure interaction must also be accounted for. The current approach uses one-way coupling: fluid pressures are transmitted to the fuel rod, but response of the coolant to movement of the rod is not calculated. Finally, transfer errors from the fluid grid to the fuel rod grid must be controlled [2], and the impact of the resulting errors in the fuel rod's vibrational response should be estimated.

Uncertainty in constitutive relations contributes to uncertainty in the rod's vibrational behavior. Parameters in empirical models [10] should be treated as stochastic. This will need to be revisited as we integrate to longer times and account for phenomena such as cladding creep, pellet-cladding interaction, hydrogen uptake, and oxidation that play important roles over longer time frames. Micro-structural simulations can help to inform the empirical constitutive models and develop confidence in the parametrization of the constitutive model.

\footnotetext{
${ }^{1}$ Note that most of the GTRF data sources identified in [3] are concerned with cladding behavior.
} 
These details are needed for both the rod material and the spacer grid surfaces (springs and dimples).

The wear model that is used in CASL's GTRF simulations is a work-rate model proposed in [7]. This model determines the wear profile by integrating over time the product of a wear coefficient, the shear traction, and the tangential velocity in the direction of the shear traction. As noted above, uncertainty that is propagated to the shear traction and tangential velocities should be included when analyzing wear depth uncertainty. The contribution to uncertainty from the friction coefficient should also be included. Fortunately this can measured independently, e.g. [19], but note that here, too, the supporting data is somewhat sparse. The wear coefficient has been observed experimentally to vary with the normal force [8]; however, there is data for only a few values of the normal force, and only a few observations for each value of the normal force. The dependence of the wear coefficient on the normal force needs to be investigated further and should also be treated as an epistemic uncertainty.

A longer-term issue that needs to be addressed for predictive simulation of GTRF is the generation of debris that becomes trapped in the contact region [5]. This debris may be the result of material removed from the surface of the fuel rod, and plays an important role in fretting fatigue. Models for material removal still need to be developed.

\section{Conclusions}

In order to be useful to decision-makers seeking to reduce the incidence of GTRF, CASL's simulations of GTRF must be accompanied by a comprehensive uncertainty analysis. The VITRAN analysis provides an excellent starting point, but CASL's VUQ efforts should apply our unique capabilities to examine joint distributions of uncertain parameters, to perform global sensitivity studies, to treat epistemic uncertainties, to perform model calibration, and to construct surrogate models. The VUQ analysis that was planned for this discontinued VUQ effort identified maximum wear depth (at both dimples and springs) as the quantity of interest, but would only have addressed the dependence of the maximum wear depth on the least important uncertain parameters. Moving forward, it is essential that new GTRF simulation capabilities are structured to facilitate uncertainty analysis. In particular, to address the most important parameters identified in the VITRAN studies [14, 16], grid-to-rod gaps that are representative of all phases of reactor operation should be available and must be treatable as epistemic uncertainties, and stochastic characterization of the fluid pressure boundary conditions needs to be developed.

\section{References}

[1] R. Buechel, Z. Karoutas, and R. Lu. Grid to rod fretting performance of Westinghouse fuel, 2008. Proceedings of the 2008 Water Reactor Fuel 
Performance Meeting.

[2] K. Copps. Verification of the coupled fluid/solid transfer in a CASL grid-torod fretting simulation. Technical Report SAND2011-9153, Sandia National Laboratories, 2011.

[3] N. Dinh. CASL validation data: An initial review. Technical Report INL/EXT-11-21017, Idaho National Laboratory, 2011.

[4] A. Elmahdi, R. Lu, M. Conner, Z. Karoutas, and E. Baglietto. Flowinduced vibration forces on a fuel rod by LES CFD analysis, 2011. Proceedings of the 14th International Topical Meeting on Nuclear Reactor Thermal Hydraulics (NURETH14).

[5] A. Hayrapetian and M. Demkowicz. Available microstructural based modeling framework of clad/grid contact, 2011. Attachment A, CASL L3:MPO.GTRF.P2.05 Milestone Report.

[6] A. Hayrapetian, M. Demkowicz, and P. Blau. Data models of wear and tribology relevant for GTRF problem, 2011. Attachment B, CASL L3:MPO.GTRF.P2.05 Milestone Report.

[7] A. Hayrapetian, M. Demkowicz, and P. Blau. Initial wear model for vi implementation for GTRF challenge problem, 2011. Attachment C, CASL L3:MPO.GTRF.P2.05 Milestone Report.

[8] K.-T. Kim and Y.-H. Lee. Wear depth model for thin tubes with supports. Wear, 263:532-541, 2007.

[9] K.-T. Kim and J.-M. Suh. Impact of nuclear fuel assembly design on grid-torod fretting wear. Journal of Nuclear Science and Technology, 46:149-157, 2009 .

[10] M. Le Saux, J. Besson, S. Carassou, C. Poussard, and X. Averty. A model to describe the anisotropic viscoplastic mechanical behavior of fresh and irradiated zircaloy-4 fuel claddings under RIA loading conditions. Journal of Materials, 378:60-69, 2008.

[11] R. Lu, Z. Karoutas, and T.-L.Sham. CASL virtual reactor predictive simulation: Grid-to-rod fretting wear. Journal of Materials, 63:53-58, 2011.

[12] W. Rider and J. Kamm. Advanced solution verification of CFD solutions for LES of relevance to GTRF estimates. Technical Report SAND20127199, Sandia National Laboratories, 2012.

[13] S. Rodriguez and D. Turner. Assessment of existing Sierra/Fuego capabilities related to grid-to-rod-fretting (GTRF). Technical Report SAND20120530, Sandia National Laboratories, 2011.

[14] P. Rubiolo. Probabilistic prediction of fretting-wear damage of nuclear fuel rods. Nuclear Engineering and Design, 236:1628-1640, 2006. 
[15] P. Rubiolo and M. Young. VITRAN: An advanced statistic tool to evaluate fretting wear damage. Journal of Power and Energy Systems, 2:57-66, 2008.

[16] P. Rubiolo and M. Young. On the factors affecting the fretting-wear risk of PWR fuel assemblies. Nuclear Engineering and Design, 239:68-79, 2009.

[17] J. Shadid, T. Smith, R. Pawlowksi, E. Cyr, P. Weber, and D. Turner. Updated initial Drekar LES CFD simulations for grid-to-rod fretting. Technical Report SAND2012-XXXX, Sandia National Laboratories, 2012.

[18] T.-L. Sham, N. Crane, P. Nath, Z. Hu, D. Turner, T. Li, and R. Sankaran. Three-dimensional, high resolution, coupled fluid/materials demonstration and assessment of grid-to-rod fretting (GTRF) test problem. Technical Report ORNL/TM-2012/3, Oak Ridge National Laboratory, 2012.

[19] G. Sharma, P. Lamaye, and D. Jadhav. Sliding wear and friction behaviour of zircaloy-4 in water. Journal of Nuclear Materials, 394:151-154, 2009.

[20] J. Stewart and H. C. Edwards. A framework approach for developing parallel adaptive multiphysics applications. Finite Elements in Analysis and Design, 40:1599-1617, 2004. 